\title{
Isotopic Studies for Tracking Biogenic Carbon during Co- processing of Biomass and Vacuum Gas Oil
}

Calvin Mukarakate, ${ }^{\text {a }}$ Kellene Orton, ${ }^{\mathrm{a}}$ Yeonjoon Kim, ${ }^{\mathrm{a}}$ Stefano Dell'Orco, ${ }^{\mathrm{b}}$ Carrie A. Farberow, ${ }^{\mathrm{a}}$ Seonah Kim, ${ }^{a}$ Michael J. Watson, ${ }^{\mathrm{c}}$ Robert M. Baldwin ${ }^{\mathrm{a}}$ and Kimberly A. Magrini ${ }^{\mathrm{a}}$

aNational Renewable Energy Laboratory, 15523 Denver West Parkway, Golden, CO 80401-3393, USA.

${ }^{b}$ Department of Industrial Engineering, University of Florence, Italy

cJohnson Matthey Technology Centre, PO Box 1, Belasis Avenue, Billingham, Cleveland TS23 1LB, UK

Corresponding aouthor: Calvin.Mukarakate@nrel.gov

Number of pages: $\quad 9$

Number of Figures: $\quad 8$ 


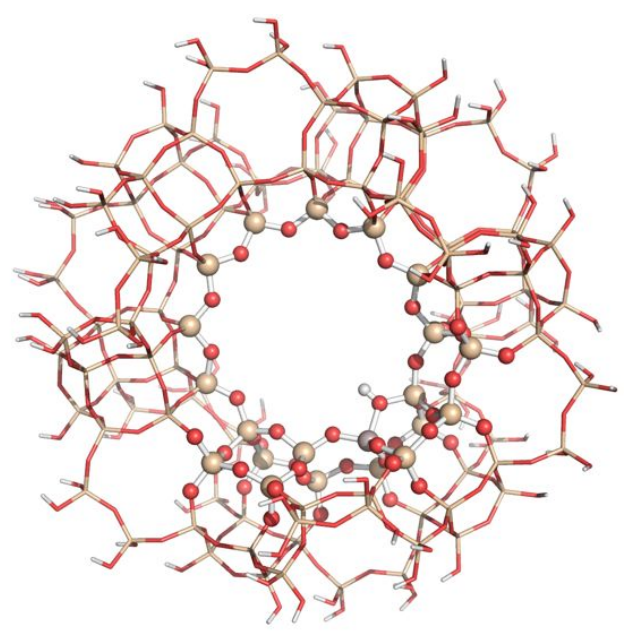

Figure S1. The HY zeolite cluster modelled in this study. Atoms depicted in ball-and-stick representation are in the high-level layer and treated using DFT, and the remaining atoms depicted using a wireframe representation were calculated using the PM6 semi-empirical method. For clarity, substrate molecules are not shown in this figure. 


\section{A) E-cat}

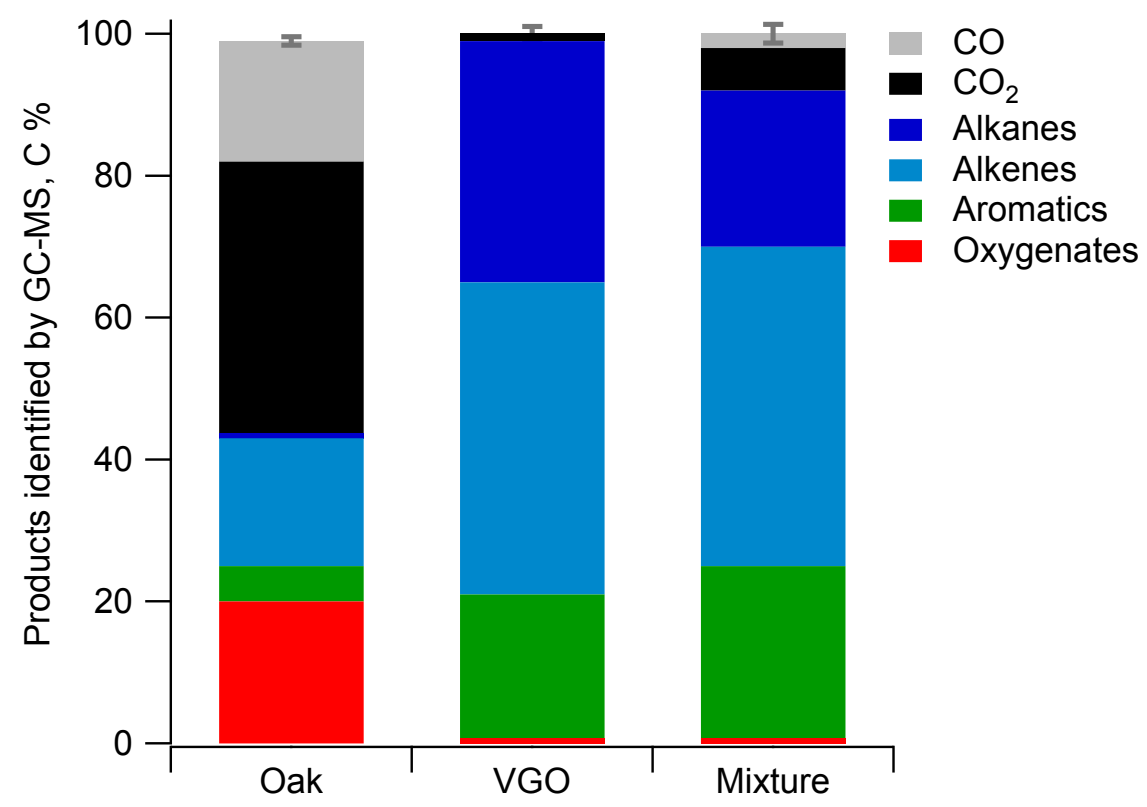

B) $\mathbf{C P 7 5 8}$

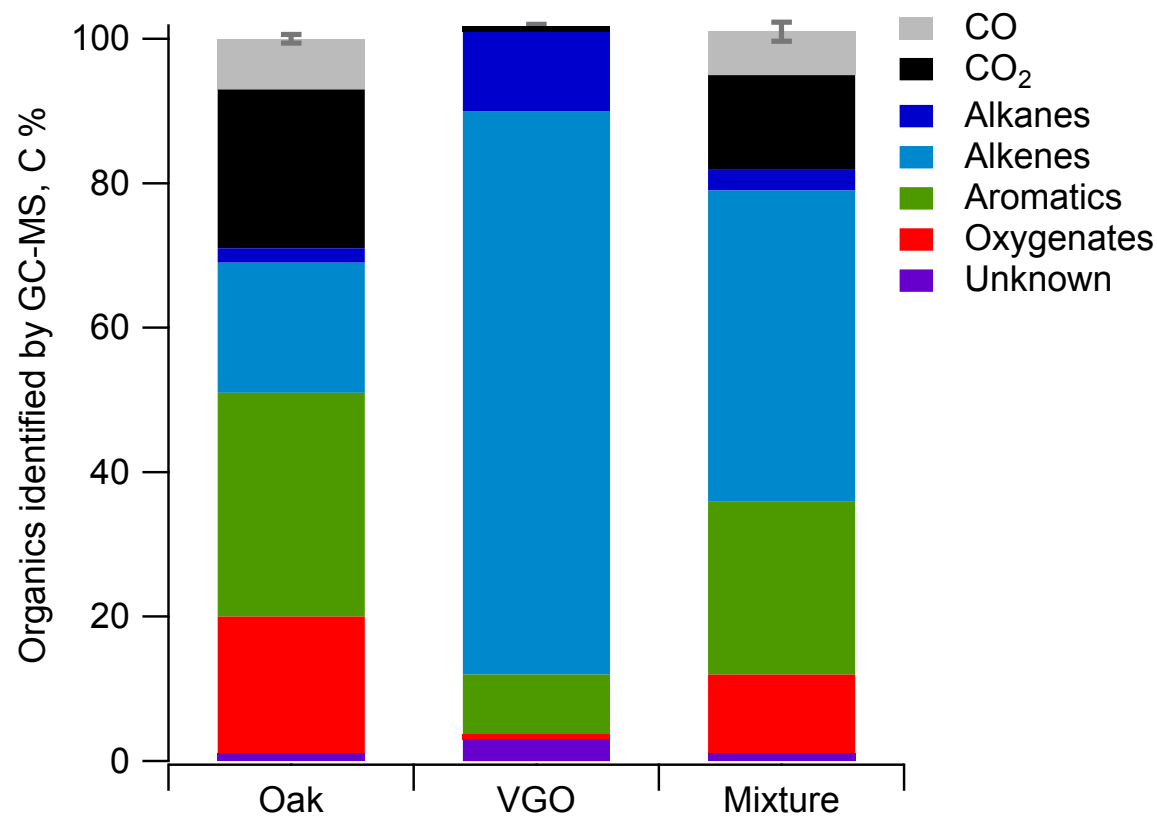

Figure S2. Catalytic cracking of VGO, oak and Oak-VGO mixture over A) E-cat, and B) CP758 at 550 ${ }^{\circ} \mathrm{C}$. Identified compounds account for $25 \mathrm{C} \%$ (neat oak), $75 \mathrm{C} \%$ (neat VGO) and $70 \mathrm{C} \%$ (Oak-VGO mixture) for E-cat and $37 \mathrm{C} \%$ (neat oak), $35 \mathrm{C} \%$ (neat VGO) and $39 \mathrm{C} \%$ (Oak-VGO mixture) for CP758. 


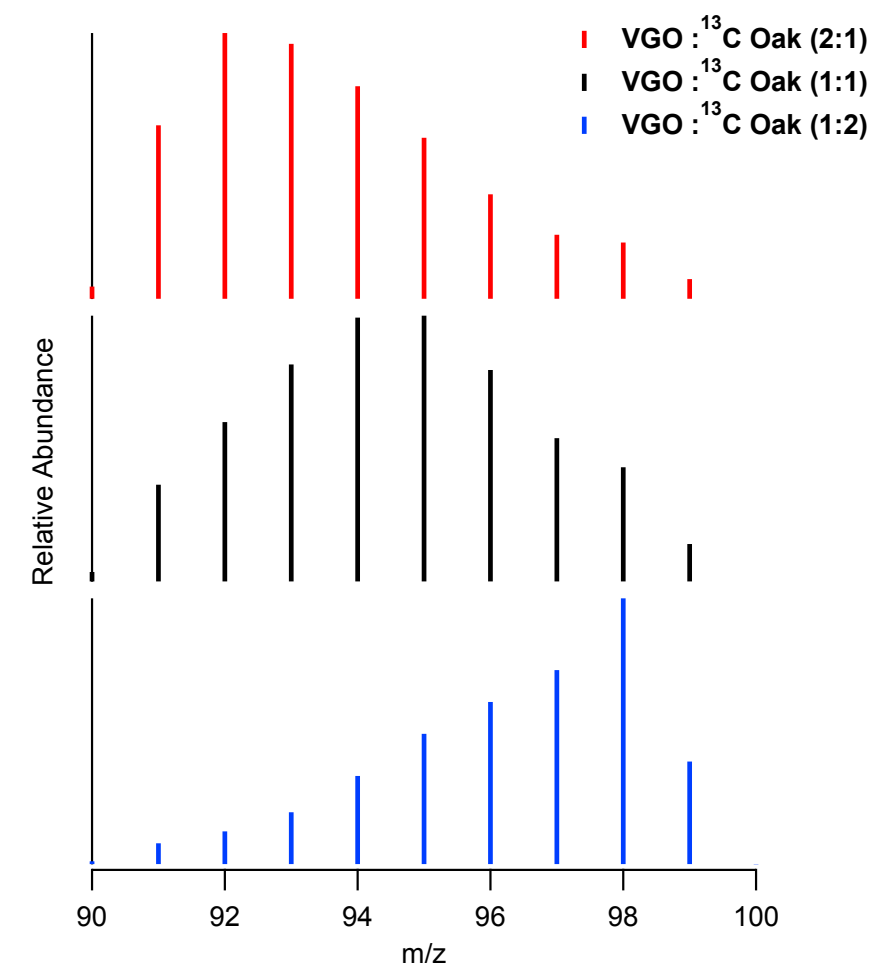

Figure S3. Biogenic carbon incorporation into $p$-xylene during co-processing of VGO and ${ }^{13} \mathrm{C}$-labelled oak over CP758 catalyst, at different feed ratios A) VGO: ${ }^{13} \mathrm{C}$ Oak $2: 1$, B) VGO: ${ }^{13} \mathrm{C}$ Oak 1:1, C) VGO: ${ }^{13} \mathrm{C}$ Oak 1:2. Reaction conditions: VGO $0.3 \mathrm{mg}$, oak $0.3 \mathrm{mg}$, catalyst $10 \mathrm{mg}$, temperature $550{ }^{\circ} \mathrm{C}$. 


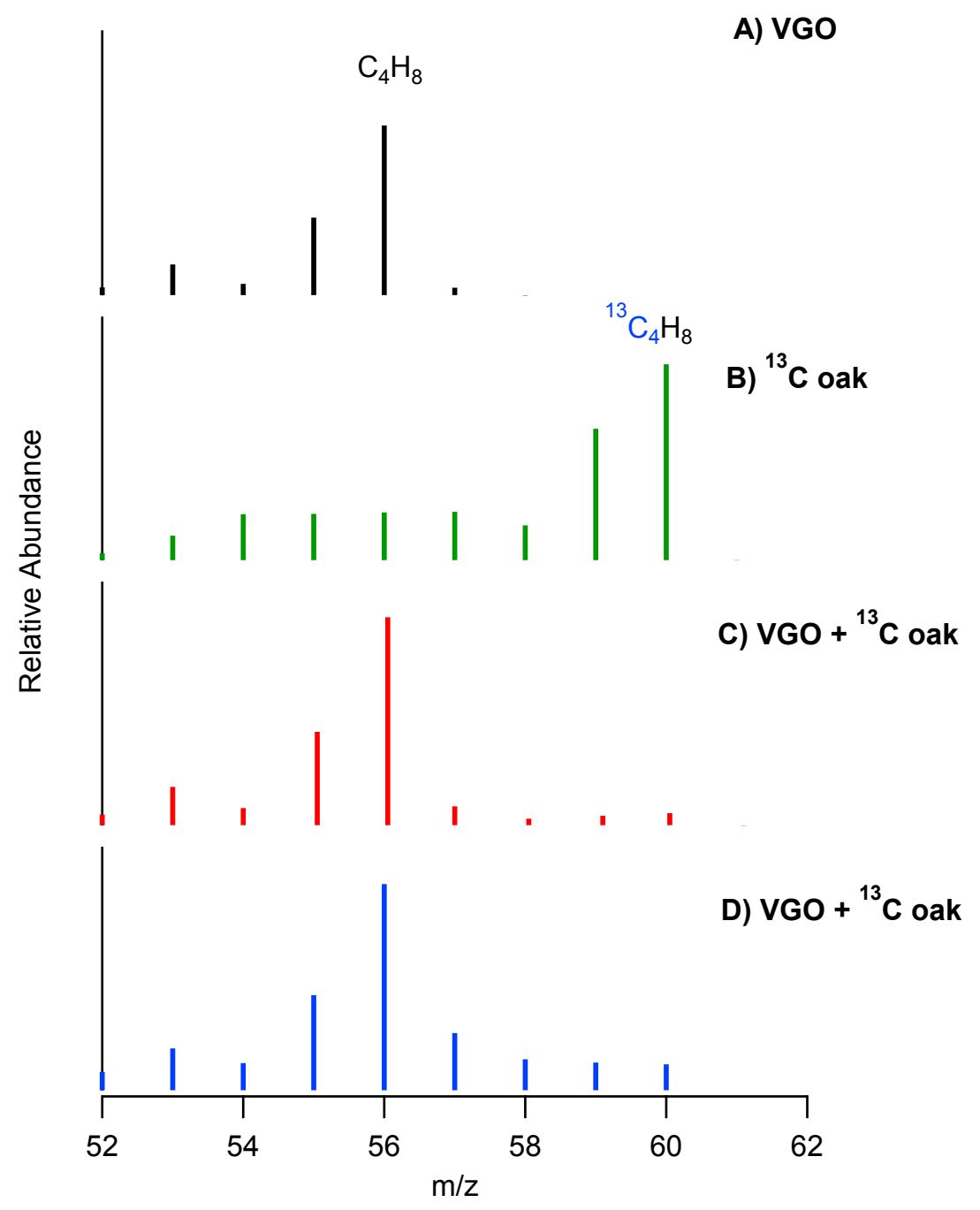

Figure S4. Biogenic carbon incorporation into methyl propene during co-processing of $\mathrm{VGO}$ and ${ }^{13} \mathrm{C}$ labelled oak, A) VGO cracking over E-cat, B) cracking ${ }^{13} \mathrm{C}$ oak over E-cat, C) cracking of VGO- ${ }^{13} \mathrm{C}$ Oak mixture over E-cat, and D) cracking of $\mathrm{VGO}-{ }^{13} \mathrm{C}$ Oak mixture over CP758. Reaction conditions: VGO $0.3 \mathrm{mg}$, oak $0.3 \mathrm{mg}$, catalyst $10 \mathrm{mg}$, temperature $550{ }^{\circ} \mathrm{C}$. 


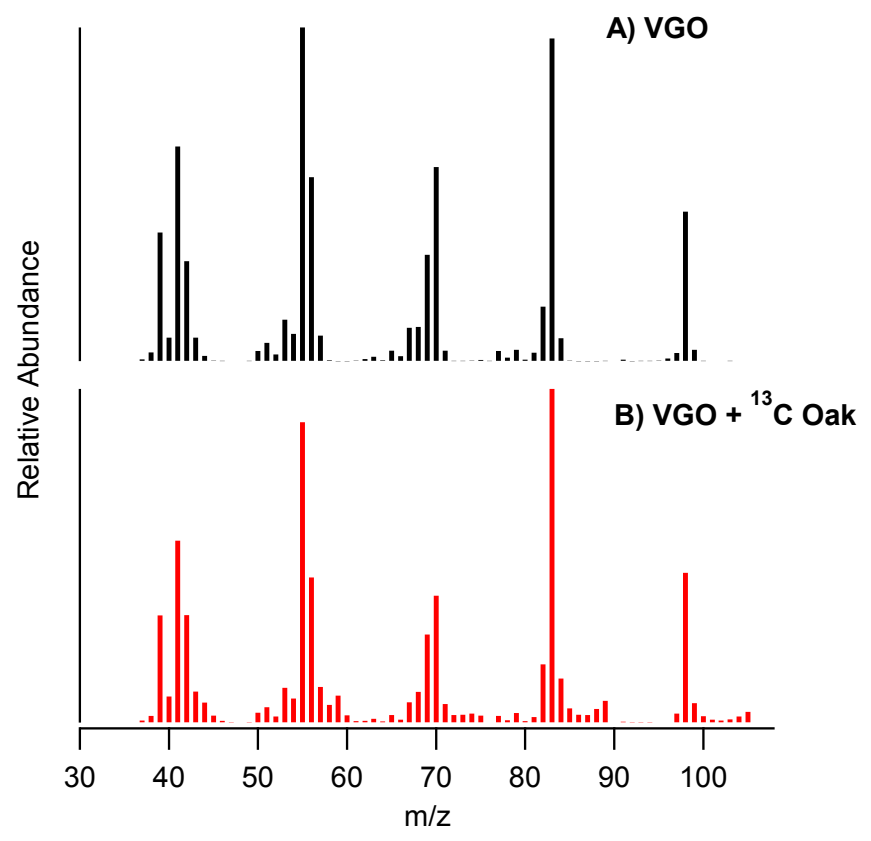

Figure S5. Biogenic carbon incorporation into methyl cyclohexane during co-processing of VGO and ${ }^{13} \mathrm{C}$ labeled oak, A) VGO cracking over E-cat, and B) cracking of VGO- ${ }^{13} \mathrm{C}$ Oak mixture over E-cat. Reaction conditions: VGO $0.3 \mathrm{mg}$, oak $0.3 \mathrm{mg}$, catalyst $10 \mathrm{mg}$, temperature $550{ }^{\circ} \mathrm{C}$. 


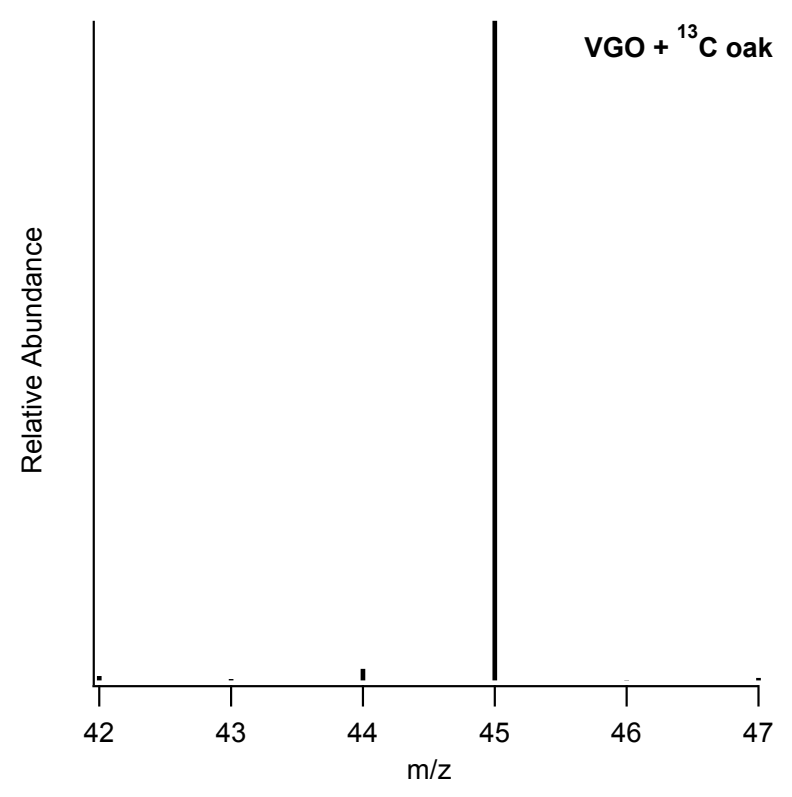

A)

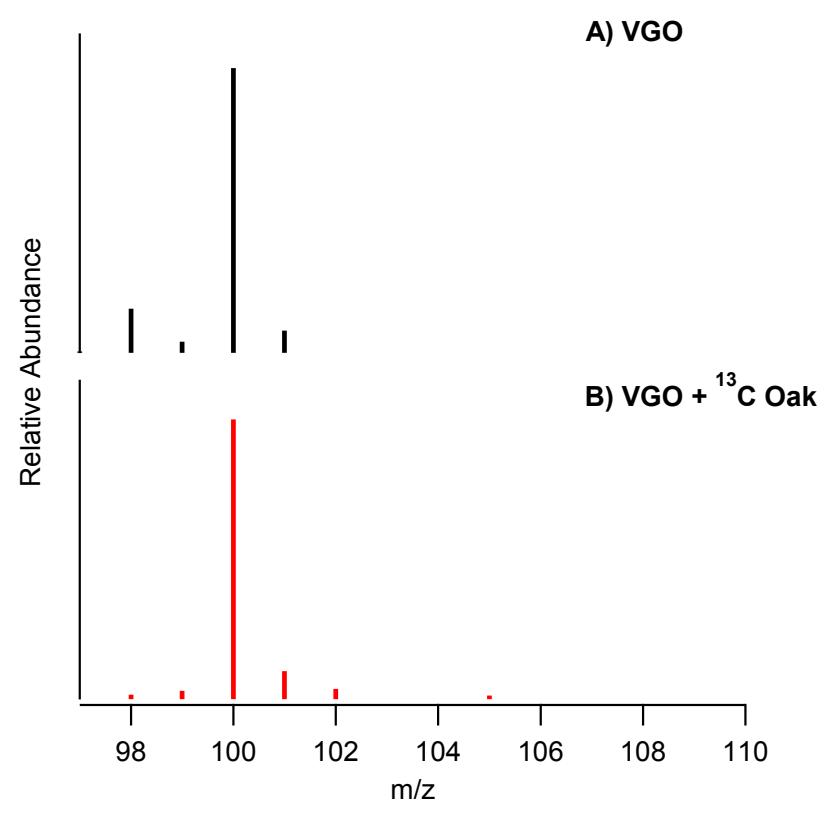

B)

Figure S6. Biogenic carbon incorporation into A) $\mathrm{CO}_{2}$ and B) 3-methyl hexane during co-processing of VGO and ${ }^{13} \mathrm{C}$-labeled oak over E-cat. Reaction conditions: VGO $0.3 \mathrm{mg}$, oak $0.3 \mathrm{mg}$, catalyst $10 \mathrm{mg}$, temperature $550{ }^{\circ} \mathrm{C}$. 

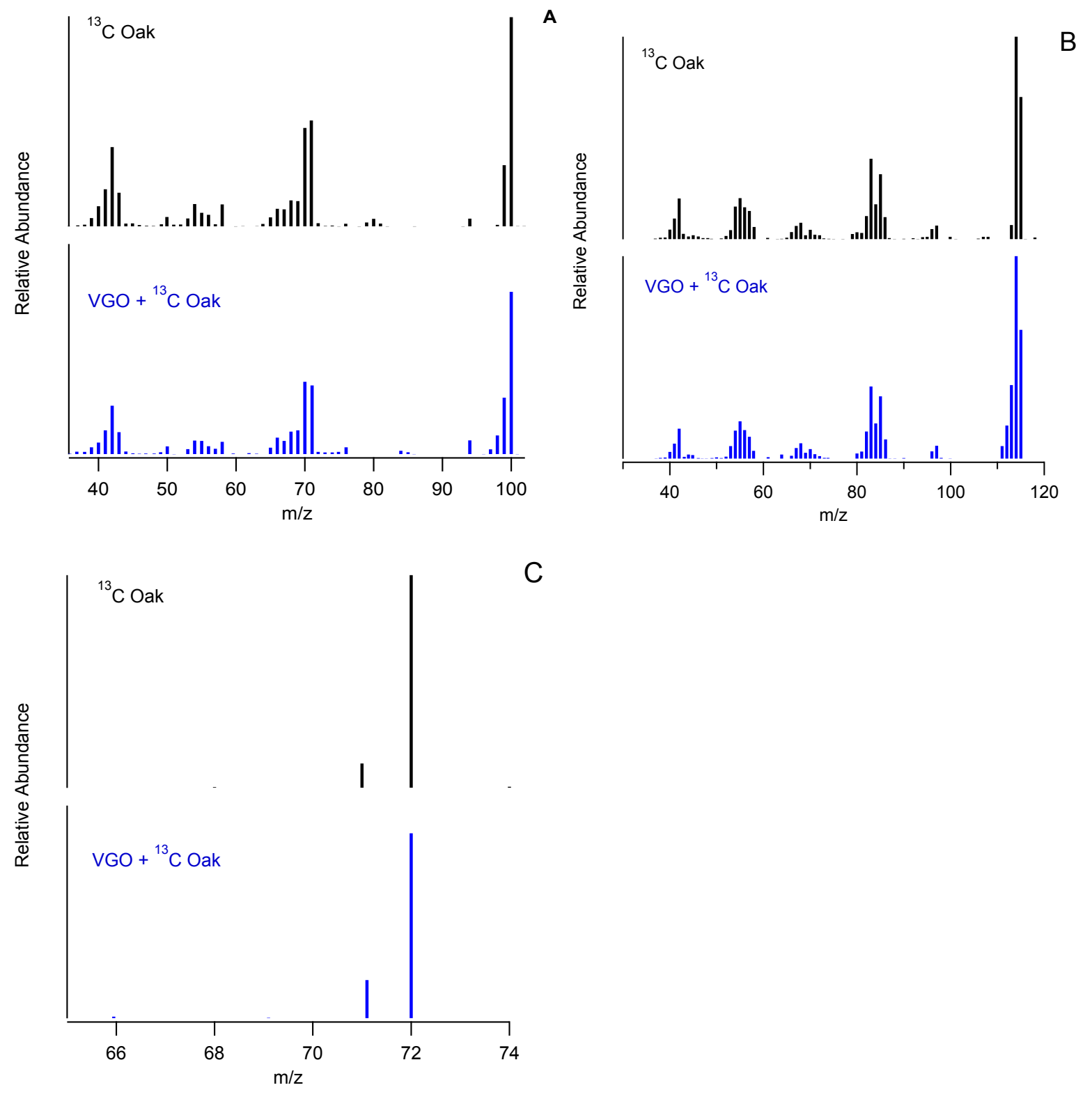

Figure S7. Fossil carbon incorporation into biomass derived molecules during coprocessing of VGO and ${ }^{13} \mathrm{C}$-labeled oak over CP758, A) phenol, B) 4-methylphenol and C) furan. Reaction conditions: VGO $0.3 \mathrm{mg}$, oak $0.3 \mathrm{mg}$, catalyst $10 \mathrm{mg}$, temperature $550{ }^{\circ} \mathrm{C}$. 


\section{A) Oak}

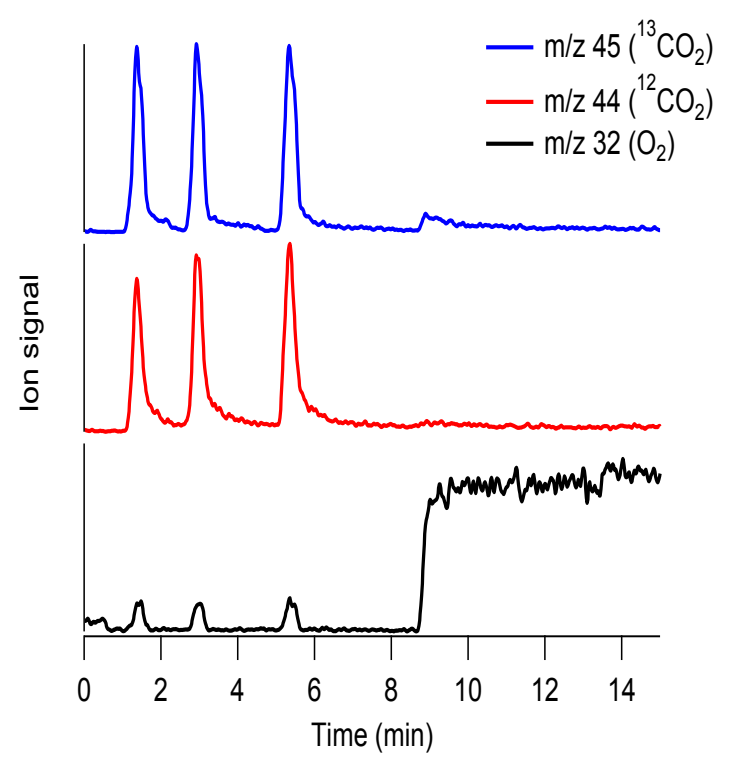

B) VGO

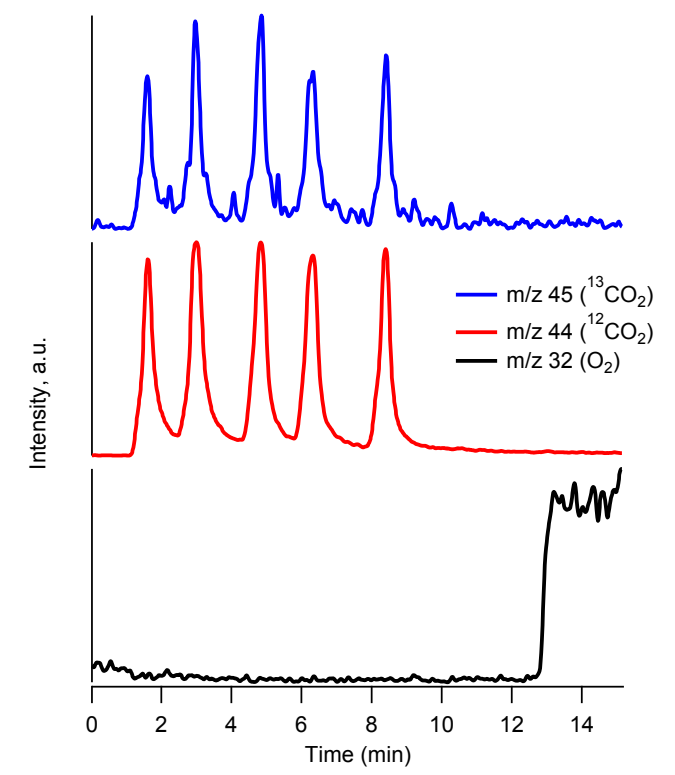

Figure S8. Ion signals of products from catalytic upgrading of A) ${ }^{13} \mathrm{C}$ oak over E-cat and B) VGO over Ecat followed by catalyst regeneration in the py-MBMS. Reaction conditions: VGO 30mg, oak 30mg, per pulse catalyst $500 \mathrm{mg}$, temperature $550{ }^{\circ} \mathrm{C}$. 